Original Paper http://ajol.info/index.php/ijbcs http://indexmedicus.afro.who.int

\title{
Intérêt du sang veineux de cordon ombilical dans le diagnostic de l'hypoglycémie néonatale
}

\author{
Youzan Ferdinand DJOHAN ${ }^{1 *}$, Massara CISSE-CAMARA ${ }^{1}$, Amenan Germeine \\ NIAMKE ${ }^{1}$, Gervais KOFFI ${ }^{1}$, Luc DERE ${ }^{2}$, Absalome Aké MONDE ${ }^{1}$, Georges TIAHOU², \\ Prosper DJESSOU ${ }^{1}$ et Daniel SESS ${ }^{1}$ \\ ${ }^{1}$ Laboratoire de Biochimie Médicale, UFR Sciences Médicales, Université Félix Houphouet-Boigny de Cocody, \\ Abidjan, Côte d'Ivoire. \\ ${ }^{2}$ Laboratoire de Biochimie Médicale, UFR Sciences Médicales, Université Alassane Ouattara de Bouaké, \\ Côte d'Ivoire. \\ *Auteur correspondant, E-mail : djohanferdinand@gmail.com, Tél: 0022503227805
}

\section{RESUME}

La confirmation de l'hypoglycémie néonatale au laboratoire nécessite du sang veineux. Devant les difficultés de prélèvement chez les nouveau-nés, une alternative s'impose. L'objectif de l'étude était d'apprécier l'intérêt du sang de cordon dans le diagnostic de l'hypoglycémie néonatale. Il s'agissait d'une étude transversale réalisée chez 150 nouveau-nés. Ont été inclus dans l'étude tous les nouveau-nés des deux sexes, nés par voie basse ou par césarienne. Chaque nouveau-né a bénéficié de deux prélèvements de sang, au talon et cordon. En salle d'accouchement, dix microlitres du sang de cordon et le sang capillaire ont servi à doser la glycémie avec un glucomètre. Au Laboratoire de Biochimie, le sang de cordon a servi à doser la glycémie par la méthode enzymatique à la glucose oxydase. Il ressort au terme de l'étude que : le plus fort taux d'hypoglycémie $(12,67 \%)$ a été obtenu avec le sang de cordon ; la différence observée entre les hypoglycémies au cordon et capillaires n'était pas statistiquement significative $(\mathrm{p}=0,58)$; les glycémies au cordon étaient bien corrélées avec les glycémies capillaires $(r=0,98)$. Devant les difficultés de prélèvement de sang veineux chez le nouveau-né, le sang de cordon apparaît comme une alternative fiable pour la confirmation de l'hypoglycémie.

(C) 2015 International Formulae Group. All rights reserved.

Mots clés : Hypoglycémie, nouveau-né, diagnostic, cordon ombilical, glucomètre.

\section{Interest of venous umbilical cord blood in the diagnosis of neonatal hypoglycemia}

\begin{abstract}
Confirmation of neonatal hypoglycemia in the laboratory requires venous blood. In front of the sampling difficulties in newborns, an alternative is needed. The objective of the study was to evaluate the benefits of cord blood in the diagnosis of neonatal hypoglycemia. This was a cross-sectional study in 150 newborns. Were included in the study all the newborns of both sexes born vaginally or by caesarean section.
\end{abstract}


Two samples of blood were taken from each new born at the level of the heel and the cord. In the delivery room, ten microliters of cord blood and capillary blood was used to assay the glucose with a glucometer. The Laboratory of Biochemistry, cord blood was used to assay the glucose by enzymatic method with glucose oxidase. It appears at the end of the study that: the highest rates of hypoglycemia (12.67\%) was obtained with the cord blood; the observed difference between the cord and hair hypoglycemia was not statistically significant $(\mathrm{p}=0.58)$; blood glucose in the cord correlated well with capillary blood glucose $(\mathrm{r}=0.98)$. In front of the venous blood sampling difficulties in the newborn, the cord blood appears to be a reliable alternative for confirmation of hypoglycemia.

(C) 2015 International Formulae Group. All rights reserved.

Keywords: Hypoglycemia, newborn, diagnosis, umbilical cord, glucometer.

\section{INTRODUCTION}

L'hypoglycémie néonatale est la baisse de la glycémie chez le nouveau-né. Le seuil de la glycémie retenu pour définir l'hypoglycémie reste variable selon les praticiens (McGowan, 2004). Devant cet état de fait, Cornblath et al. (2000) proposent la valeur seuil de $2,6 \mathrm{mmol} / \mathrm{l}$ pour définir l'hypoglycémie néonatale. Le glucose est la principale source d'énergie pour le cerveau du foetus et du nouveau-né. Non dépisté à temps, l'hypoglycémie néonatale aura comme conséquence grave, une atteinte neurologique irréversible (Menni et al., 2001; Boardman et al., 2013).Ce faisant, l'hypoglycémie demeure une urgence diagnostique et thérapeutique. Le diagnostic de l'hypoglycémie néonatale commence en salle d'accouchement par le dépistage avec un glucomètre sur du sang capillaire, et doit être confirmée au laboratoire sur du sang veineux du nouveau-né (Sperling et Menon, 2004).

L'étude de la structure physiologique du cordon ombilical, permet de comprendre que le sang circulant dans le cordon est identique à celui circulant chez le nouveau-né. Partant de ce principe, des examens tels que le dosage du lactate (Linet et al., 2002), le groupage sanguin (Gallet et al., 2002) peuvent être réalisés sur sang de cordon. Compte tenu de la difficulté des prélèvements veineux chez les nouveau-nés pour confirmer l'hypoglycémie néonatale, le sang de cordon ombilical a été utilisé.

L'objectif de cette étude était de voir si les glycémies dosées au laboratoire sur sang de cordon étaient superposables aux glycémies capillaires obtenues en salle d'accouchement chez les nouveau-nés.

\section{MATERIEL ET METHODES \\ Patients}

Cette étude transversale a été réalisée au Laboratoire de Biochimie de l'Unité de Formation et de Recherche des Sciences Médicales d'Abidjan (UFRSMA) en collaboration avec le service de gynécologie $\mathrm{du}$ Centre Hospitalier Universitaire de Treichville. Elle a portée sur 150 nouveau-nés sélectionnés en salle d'accouchement. Ont été inclus dans l'étude tous les nouveau-nés (prématurés et à terme) des deux sexes, nés en salle d'accouchement, par voie basse ou par césarienne. N'ont pas été inclus dans l'étude les enfants nés hors de la salle d'accouchement. Le protocole de l'étude a été approuvé par l'Unité de Formation et de Recherche des Sciences Médicales de l'Université Félix Houphouët-Boigny de Cocody, et a été expliqué aux parents des nouveau-nés avant qu'ils aient donné leur consentement éclairé.

\section{Prélèvements}

Deux prélèvements de sang ont été effectués chez chaque nouveau-né dix minutes après sa naissance. Un prélèvement capillaire au talon et un prélèvement de sang veineux au cordon ombilical. Le sang de cordon a été recueilli dans un tube à bouchon gris (BD vacutainer ${ }^{\circledR}$ ) contenant du fluorure de sodium et de l'oxalate de potassium. Après avoir été soigneusement homogénéisé, le sang du cordon a été rapidement acheminé au Laboratoire de Biochimie pour le dosage de la glycémie. 


\section{Dosage}

En salle d'accouchement, $10 \mu \mathrm{l}$ du sang veineux de cordon et de sang capillaire recueilli au talon ont servi au dosage de la glycémie avec le glucomètre One Touch Ultra $^{\circledR}($ OTU) de Lifescan. Au Laboratoire de Biochimie, le sang a été centrifugé à 3000 rpm pendant 5 minutes. Le plasma obtenu a servi au dosage de la glycémie par la méthode enzymatique à la glucose oxydase (E1102) avec un automate multiparamétrique (Roche, Hitachi $912^{\circledR}$ ).

\section{Analyses statistiques}

Le traitement des données recueillies a été réalisé à l'aide du logiciel Epi-info version 6.0. Les résultats ont été exprimés en moyenne \pm écart type. Le coefficient de corrélation de Pearson a été utilisé pour déterminer le degré de corrélation des glycémies capillaire et veineuse. La comparaison des moyennes a été réalisée par le test paramétrique de Student. La différence a été considérée comme statistiquement significative lorsque $\mathrm{p}<0,05$.

\section{RESULTATS}

Les résultats de cette étude ont été consignés dans les Tableaux 1 et 2 et les Figures 1 et 2 . Il ressort que les différences observées entre les moyennes des glycémies obtenues par les différentes techniques sont statistiquement significative $\quad(p<0,05)$ (Tableau 1). Le plus fort taux d'hypoglycémie $(12,67 \%)$ a été obtenu au laboratoire avec le sang de cordon (Tableau 2). La différence observée entre les taux d'hypoglycémies décelées au laboratoire sur le sang de cordon et en salle d'accouchement sur le sang capillaire n'était pas statistiquement significative $(p=0,58)$ (Tableau 2). Les glycémies au cordon dosées au laboratoire étaient bien corrélées avec les glycémies capillaires $(r=0,98)$ (Figure 1), tandis que les glycémies au cordon avec le glucomètre OTU étaient moins corrélées avec les glycémies capillaires $(r=0,76)$ (Figure 2).

Tableau 1 : Comparaison des valeurs moyennes des glycémies obtenues avec l'Hitachi 912 et l'OTU.

\begin{tabular}{lccc}
\hline & $\begin{array}{c}\text { Moyenne } \pm \text { et } \\
(\mathbf{m m o l} / \mathbf{l})\end{array}$ & Comparaison & Valeur de $\boldsymbol{p}$ \\
\hline Hitachi $912_{\mathrm{C}}$ & $5,06 \pm 1,41$ & Hitachi $912_{\mathrm{C}} V s$ OTU & \\
OTU $_{\mathrm{C}}$ & $5,44 \pm 1,43$ & OTU $_{\mathrm{C}} V s$ OTU & 0,026 \\
OTU $_{\mathrm{T}}$ & $4,56 \pm 1,23$ & OTU $_{\mathrm{T}} V s$ Hitachi $912_{\mathrm{C}}$ & 0,000 \\
\hline${\text { Hitachi } 912_{\mathrm{C}}: \text { Glycémie obtenue au laboratoire sur sang veineux du cordon ombilical avec Hitachi } 912 .}$ \\
OTU $_{\mathrm{C}}:$ Glycémie obtenue en salle d'accouchement sur sang veineux du cordon ombilical avec le glucomètre OTU. \\
OTU $_{\mathrm{T}}:$ Glycémie obtenue en salle d'accouchement sur sang capillaire au talon avec le glucomètre OTU.
\end{tabular}

Tableau 2 : Comparaison des taux d'hypoglycémies décelées par l'Hitachi 912 et l'OTU.

\begin{tabular}{ccccc}
\hline & $\begin{array}{c}\text { Nombre } \\
\text { d'hypoglycémies } \\
\text { décelées }\end{array}$ & Pourcentage $(\%)$ & Comparaison & Valeur de $\boldsymbol{p}$ \\
\hline Hitachi $912_{\mathrm{C}}$ & 19 & $12,67(19 / 150)$ & $\begin{array}{c}\text { Hitachi } 912_{\mathrm{C}} V s \\
\text { OTU }_{\mathrm{C}}\end{array}$ & 0,000 \\
OTU $_{\mathrm{C}}$ & 10 & $6,67(10 / 150)$ & $\begin{array}{c}\text { OTU } \\
\text { OTU } \text { OTU }_{\mathrm{T}}\end{array}$ & 0,019 \\
OTU $_{\mathrm{T}}$ & 17 & $11,33(17 / 150)$ & $\begin{array}{c}\text { OTU } \\
\text { Hitachi } 912_{\mathrm{C}}\end{array}$ & 0,58 \\
\hline
\end{tabular}

Hitachi 912 : Glycémie obtenue au laboratoire sur sang veineux du cordon ombilical avec Hitachi 912.

OTU $_{C}$ : Glycémie obtenue en salle d'accouchement sur sang veineux du cordon ombilical avec le glucomètre OTU.

$\mathrm{OTU}_{\mathrm{T}}$ : Glycémie obtenue en salle d'accouchement sur sang capillaire au talon avec le glucomètre OTU. 


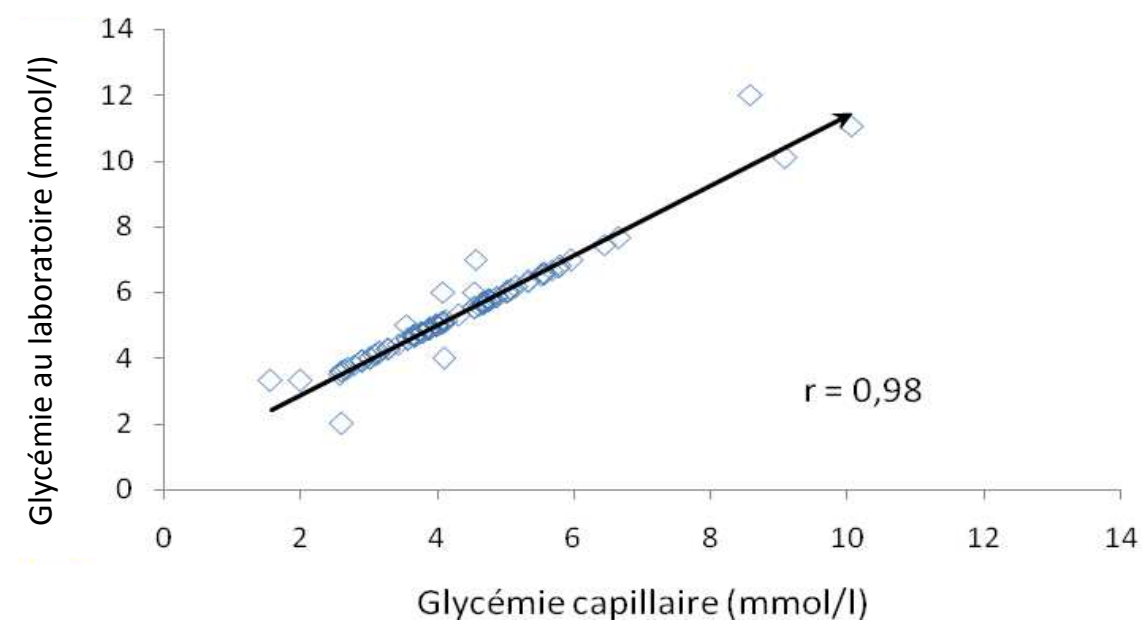

Figure 1 : Corrélation entre les glycémies capillaires et les glycémies dosées au laboratoire.

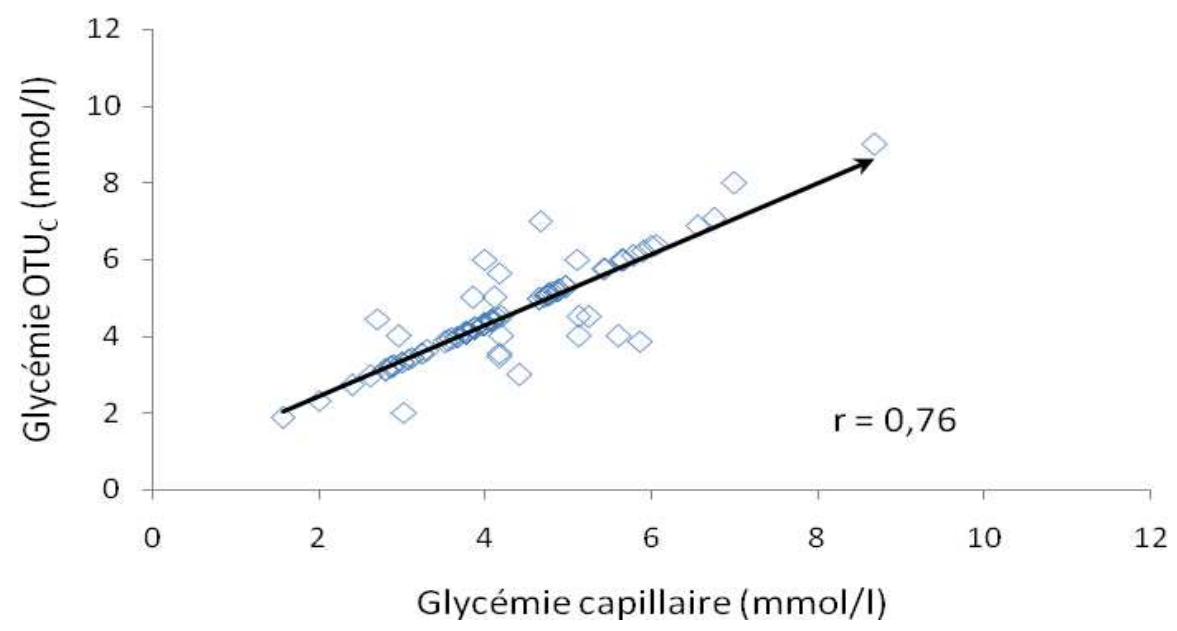

Figure 2 : Corrélation entre les glycémies capillaires et les glycémies au cordon avec OTU. OTU ${ }_{\mathrm{C}}$ : Glycémie obtenue en salle d'accouchement sur sang veineux du cordon ombilical avec le glucomètre OTU.

\section{DISCUSSION}

La moyenne des glycémies obtenues était de $5,06 \mathrm{mmol} / \mathrm{l}$ pour l'Hitachi $912_{\mathrm{C}}$, de $5,44 \mathrm{mmol} / 1$ pour l'OTU $\mathrm{C}_{\mathrm{C}}$ et de $4,56 \mathrm{mmol} / \mathrm{l}$ pour l'OTU $\mathrm{T}_{\mathrm{T}}$. La comparaison entre ces valeurs montre que les glycémies obtenues avec le glucomètre OTU sur du sang de cordon ombilical $\left(\mathrm{OTU}_{\mathrm{C}}\right)$ sont significativement plus élevées que celles obtenues d'une part au laboratoire avec l'Hitachi $912_{\mathrm{C}}$ sur le même prélèvement $(\mathrm{p}=0,026)$ et d'autre part en salle d'accouchement sur le sang capillaire au talon $\left(\mathrm{OTU}_{\mathrm{T}}\right)$ avec $\mathrm{p}=0,000$ (Tableau 1). La glycémie capillaire étant en moyenne inférieure de 10 à $15 \%$ à celle mesurée sur plasma veineux (Funk et al., 2001; Kuwa et al., 2001; Ho et al., 2004), les glucomètres, de nos jours, intègrent une option de correction automatique pour faciliter la comparaison avec les résultats du laboratoire. Cette correction automatique opérée sur le sang 
veineux $\mathrm{du}$ cordon expliquerait l'augmentation des glycémies obtenues avec $\mathrm{OTU}_{\mathrm{C}}$. Cette surestimation de la glycémie veineuse par le glucomètre a été mise en évidence par de nombreux auteurs (Michel et al., 2005; Karon et al., 2007; Tonyushkina et Nichols, 2009; Nuntnarumit et al., 2011).

L'hypoglycémie néonatale entraine des lésions neuronales dont le risque de survenue augmente avec des valeurs de glycémie en dessous de 2,2 mmol/l (Cornblath et Ichord, 2000; Alkalay et al., 2005; Boluyt et al., 2006). La définition de l'hypoglycémie néonatale reste un sujet de controverse, en particulier en raison de l'absence de corrélation significative entre la glycémie, les signes cliniques, et les séquelles à long terme (Cornblath et al., 2000; Rozance et Hay, 2010). Bien que Cornblath et al. (2000) aient retenu la valeur seuil de 2,6 mmol/l pour définir l'hypoglycémie néonatale, un état des lieux a montré que la définition de l'hypoglycémie néonatale variait selon les praticiens avec des valeurs se situant entre 1,0 $\mathrm{mmol} / \mathrm{l}$ et 4,0 mmol/1 (Hay et al., 2009). Fort de tout ce qui précède, toute glycémie inférieure à 3,33 $\mathrm{mmol} / \mathrm{l}$ a été considérée comme hypoglycémie néonatale. Partant de cette valeur seuil, 19 hypoglycémies ont été décelées au laboratoire avec le sang de cordon, 17 hypoglycémies pour le sang capillaire avec le glucomètre et 10 hypoglycémies pour le sang de cordon avec le glucomètre (Tableau 2). Le sang de cordon a permis de déceler plus d'hypoglycémie au laboratoire qu'avec le glucomètre et la différence observée entre les pourcentages d'hypoglycémies détectés était statistiquement significative avec $\mathrm{p}=0,000$ (Tableau 2). Ceci montre que le sang veineux de cordon ombilical n'est pas indiqué pour un dépistage de l'hypoglycémie néonatale au glucomètre. Par contre, la différence observée entre les taux d'hypoglycémies décelées au laboratoire sur sang de cordon et en salle d'accouchement sur sang capillaire au talon n'était pas statistiquement significative, $\mathrm{p}=0,58$. L'hypoglycémie décelée au glucomètre doit être confirmée par un test en laboratoire avant qu'on puisse poser le diagnostic d'hypoglycémie, en particulier chez des nouveau-nés asymptomatiques (Cornblath et Ichord, 2000; Deshpande et Platt, 2005). Face aux difficultés de prélèvement du sang veineux chez certains nouveau-nés pour la confirmation de l'hypoglycémie au laboratoire, le sang de cordon se présenterait comme une alternative. Les taux d'hypoglycémie décelés dans l'étude étaient de $6,67 \%$ pour OTU $_{\mathrm{C}}, 11,33 \%$ pour OTU $\mathrm{OT}_{\mathrm{T}}$ et de $12,67 \%$ pour Hitachi $912_{\mathrm{C}}$. Face à l'absence d'une définition consensuelle de l'hypoglycémie néonatale (McGowan, 2004), il n'est pas possible d'en définir la fréquence exacte. Néanmoins, les résultats de cette étude se situent dans la fourchette de la littérature $0,4 \%$ et $20 \%$ (Hay et al., 2009; Pretty et al., 2010).

L'étude des corrélations est d'une importance capitale pour voir s'il y a une liaison entre deux méthodes données. La corrélation entre les glycémies obtenues au laboratoire sur le sang de cordon et les glycémies capillaires d'une part, et celle entre les glycémies capillaires et les glycémies au cordon avec le glucomètre d'autre partont été étudiées. Au plan statistique, l'élément discriminant de ces corrélations est le coefficient de corrélation " $r$ " qui doit être plus proche de 1. Entre les glycémies obtenues au laboratoire et les glycémies capillaires, $\mathrm{r}=$ 0,98 , contre $\mathrm{r}=0,76$ entre les glycémies capillaires et les glycémies au cordon avec le glucomètre. Il ressort que les glycémies obtenues au laboratoire sur sang de cordon ont une meilleure corrélation $(r=0,98)$ avec les glycémies capillaires obtenues avec le glucomètre One Touch Ultra. La bonne 
corrélation des glycémies de ce glucomètre avec celles du laboratoire pourrait s'expliquer par le fait que le glucomètre One Touch Ultra utilise la méthode à la glucose oxydase qui est la même que celle utilisée par le laboratoire. Ces résultats militent en faveur de l'utilisation du sang de cordon ombilical comme une alternative aux difficultés de prélèvement de sang veineux rencontrés chez de nombreux nouveau-nés pour confirmer l'hypoglycémie néonatale au laboratoire. Toutefois, il est important de tenir compte de la glycolyse anaérobie qui, après 30 minutes, donne de fausses hypoglycémies. Pour ce faire, le prélèvement au cordon doit se faire immédiatement après avoir coupé le cordon ombilical, le temps d'attente ne devant pas excéder 20 minutes (Fobker, 2014). Ce sang doit être prélevé dans un tube contenant un antiglycolytique (fluorure de sodium) et doit être soigneusement homogénéisé avant d'être acheminé au laboratoire.

\section{Conclusion}

L'utilisation du sang de cordon ombilical pour la confirmation de l'hypoglycémie chez le nouveau-né apparait comme une alternative fiable devant des difficultés de prélèvement de sang veineux chez certains nouveau-nés. Le prélèvement au cordon devrait être de plus en plus utilisé car il ne présente aucun danger ni pour le nouveau-né ni pour la mère.

\section{CONFLIT D'INTERETS}

Il n'existe aucun conflit d'intérêt entre les auteurs de ce travail.

\section{CONTRIBUTIONS DES AUTEURS}

YFD, MC-C et AGN ont contribué à la conception et la mise au point de l'étude, à l'analyse et l'interprétation des données et à la rédaction du manuscrit; $\mathrm{GK}$ et $\mathrm{LD}$ ont contribué à l'analyse et l'interprétation des données, à la lecture du manuscrit final ; AAM, GT, PD et DS ont contribué à la conception et la mise au point de l'étude ainsi qu'à la lecture du manuscrit final.

\section{REMERCIEMENTS}

Nous tenons à remercier les sagesfemmes de la salle d'accouchement ainsi que les techniciens du Laboratoire de Biochimie du CHU de Treichville pour leur précieuse collaboration.

\section{REFERENCES}

Alkalay AL, Flores-Sarnat L, Sarnat HB, Moser FG, Simmons CF. 2005. Brain imagingfindings in neonatalhypoglycemia: case report and review of 23 cases. Clin Pediatr, 44: 783-790. DOI :http://dx.doi.org/10.1177/ 000992280504400906

Boardman JP, Wusthoff CJ, Cowan FM. 2013. Hypoglycaemia and neonatalbraininjury. Arch Dis Child Educ Pract Ed., $\quad 98: \quad 2-6$. DOI :http://dx.doi.org/10.1136/archdischi ld-2012-302569

Boluyt N, van Kempen A, Offringa M. 2006. Neuro development after neonatal hypoglycemia : asystematic review and design of an optimal future study. Pediatrics, 117: 2231-2243. DOI : http://dx.doi.org/10.1542/peds.2005-1919

Cornblath M, Hawdon JM, Williams AF, Aynsley-Green A, Ward-Platt MP, Schwartz R. 2000. Controvers disregarding definition of neonatal hypoglycemia: suggested operational thresholds. Pediatrics, 105: 1141-1145. DOI :http://dx.doi.org/10.1542/peds.105. 5.1141

Cornblath M, Ichord R. 2000. Hypoglycemia in the neonate. Semin Perinatol., 24: 136149. DOI : http://dx.doi.org/10.1053/ sp.2000.6364 
Deshpande S, Platt MW. 2005. The investigation and management of neonatal hypoglycemia. Seminfetal Neonatal Med., 10(4): 351-361. DOI :http://dx.doi.org/10.1016/j.siny.200 5.04 .002

Fobker M. 2014. Stability of glucose in plasma withdifferent anticoagulants. Clin Chem. Lab. Med., 52(7): 1057-1060. DOI :http://dx.doi.org/10.1515/cclm2013-1049

Funk Dl, Chan L, Lutz N. 2001. Comparison of capillary and venous glucose measure mentsin healthy volunteers. Prehosp Emerg. Care, 5: 275-277. DOI :http://dx.doi.org/10.1080/10903120 190939788

Gallet M, Bulé-Pépin R, Omanga-Léké ML, Lorriaux C. 2002. Groupe sanguin érythrocytaire sur sang de cordon. $J$ Gynecolobstet Biol. Reprod., 31: 512. DOI :http://dx.doi.org/JGYN-09-200231-5-0368-2315-101019-ART11

Hay WW Jr, Raju TN, Higgins RD, Kalhan SC, Devaskar SU. 2009. Knowledge gaps and research needs for understanding and treating neonatal hypoglycemia: workshop report from Eunice Kennedy Shriver National Institute of Child Health and Human Development. J. Pediatr., 155(5): 612-617. DOI : http://dx.doi.org/10.1016/j.jpeds.2009.06. 044

Ho HT, Yeung WK, Young BW. 2004. Evaluation of "point of care" devices in the measurement of low glucose in neonatal practice. Arch Dis Child Fetal Neonatal Ed., $\quad$ 89: 356-359. DOI : http://dx.doi.org/10.1136/adc.2003.03354 8

Karon BS, Gandhi GY, Nuttall GA, Bryant SC, Schaff HV, McMahon MM. 2007. Accuracy of Roche Accu-Chek in form whole blood capillary, arterial, and venous glucose values in patients receiving intensive intravenous insulin therapy after cardiac surgery. Am. J. Clin. Pathol., 127 : 919-926. DOI : http://dx.doi.org/10.1309/6RFQCKAAJG KWB8M4

Kuwa K, Nakayama T, Hoshino T, Tominaga M. 2001. Relationships of glucose concentrations in capillary whole blood, venous whole blood and venous plasma. Clin. Chim. Acta, 307: 187-192. DOI : http://dx.doi.org/10.1016/S0009898(01)00426-0

Linet T, Laporte J, Gueye H, Boog G. 2002. Évaluation du bien-être néonatal par micro-dosage rapide des lactates au sang du cordon. J. Gynecol. Obstet. Biol. Reprod., 31 : 352-357. DOI :http://dx. doi.org/JGYN-06-2002-31-4-0368-2315101019-ART4

McGowan JE. 2004. Neonatal Hypoglycemia: fifty years later, the questions remain the same. Neoreviews, 5: e363-e364. DOI : http://dx.doi.org/10.1542/neo.5-9-e363

Menni F, Lonlay P, Sevin C, Touati G, Peigné C, Barbier V, Nihoul-Fékété C, Saudubray JM, Robert JJ. 2001. Neurologic Outcomes of 90 Neonates and Infants With Persistent Hyperinsulinemic Hypoglycemia. Pediatrics, 107(3): 476479.DOI:http://dx.doi.org/10.1542/peds. 1 07.3.476

Michel A, Küster H, Krebs A, Kadow I, Paul W, Nauck M. 2005. Evaluation of the Glucometer Elite XL device for screening for neonatal hypoglycemia. Eur. J. Pediatr., 164: 660-664. DOI : http://dx.doi.org/10.1007/s00431-0051733-9

Nuntnarumit P, Chittamma A, Pongmee P, Tangnoo A, Goonthon S. 2011. Clinical performance of the new glucometer in the nursery and neonatal intensive care unit. 
Pediatr. Int., 53: 218-223. DOI : http://dx.doi.org/10.1111/j.1442200X.2010.03214.x

Pretty CG, Chase JG, Le Compte A, Shaw GM, Signal M. 2010. Hypoglycemia detection in critical care using continuous glucose monitors : an in silico proof of concept analysis. J. Diabetes Sci Technol., 4: 15-24. DOI : http://dx.doi.org/10.1177/ 193229681000400103

Rozance PJ, Hay WW. 2010. Describing hypoglycemia - definition or operational threshold? Early Human Development,
86(5): $\quad$ 275-280. $\quad$ DOI : http://dx.doi.org/10.1016/j.earlhumdev.20 10.05.002

Sperling MA, Menon RK. 2004. Differential diagnosis and management of neonatal hypoglycemia. Pediatr. Clin. N. Am., 51: 703-723. DOI : http://dx.doi.org/10.1016/ j.pcl.2004.01.014

Tonyushkina K, Nichols HJ. 2009. Glucose meters : a review of technical challenges to obtaining accurate results. J. Diabetes Sci. Technol., 3: 971-980. DOI : http://dx.doi.org/10.1177/1932296809003 00446 